\section{LOS CENTROS DE ESTUDIOS LOCALES}

\author{
Alberto C. Ibáñez Pérez \\ Vocal de la Junta de Gobierno de la CECEL
}

\begin{abstract}
The creation of the CECEL centres is studied: it depending economically and organizationally on different entities, its tasks, its territorial area, its activity fields; Installations and services given.
\end{abstract}

KEY WORDS: The centres of Local Studies. Origin. Administrative dependence. Funding. Territorial area.

\section{Origen}

Los centros de estudios locales son fruto de la necesidad de conocimiento y consiguiente corriente investigadora iniciada con la llustración y, por ello, muchos de ellos herederos y continuadores de las Comisiones Provinciales de Monumentos nacidas con el objeto de salvaguardar todo lo más posible del ingente patrimonio histórico-artístico creado y custodiado por las órdenes religiosas cuyas instalaciones sufrieron los terribles efectos de las diferentes leyes desamortizadoras, tan mal realizadas. Las Comisiones Provinciales de Monumentos basaron su trabajo de salvaguarda de las obras en el conocimiento de las mismas a partir de su conocimiento. Labor que completaron y continuaron los centros de estudios locales y que explica la abundancia de los dedicados al estudio y divulgación de la historia y el arte con carácter exclusivo o preferente en su ámbito geográfico.

En el caso de los centros de estudios -CEL- que han tenido desde sus inicios una dedicación a campos de estudio no exclusivamente históricos y artísticos pueden encontrarse sus inicios, al menos desde un punto de vista conceptual y funcional, en las Sociedades Económicas de Amigos del Pais, que nacieron como resultado de la valoración del saber y de la educación como factores imprescindibles para el desarrollo de las tierras y lugares en los que se fundaron, con una misión formativa orientada a las actividades artesanales, no tanto educativa como de clara orientación

\section{THE CENTRES OF LOCAL STUDIES}

RESUMEN: Se estudia la creación de los centros de la CECEL, su dependencia administrativa económica y organizativa de diversas entidades, sus tareas, ámbitos territoriales, campos de actividad. Instalaciones y servicios que prestan.

PALABRAS CLAVE: Los Centros de Estudios Locales. Origen. Dependencia administrativa. Financiación. Ámbito territorial.

práctica. Aunque no fue éste el camino seguido por los CEL en su actividad.

Finalmente, acaso sean más abundantes los centros de estudios locales nacidos como resultado de la noble emulación, con la finalidad de hacer en su tierra y lugar lo mismo que se venía haciendo desde tiempo atrás en otros sitios y lograr unos resultados semejantes.

\section{Promotores y FUNDADORES}

La iniciativa en la creación de centros correspondió a las instituciones gubernativas locales y provinciales, como era lógico en el seno de una situación política de estricto control de las actividades públicas $y$, de manera especial, las relacionadas con el campo de la cultura y de las ideas. Este aspecto permite afirmar que fue el poder central el promotor e impulsor de centros y, mediante su inclusión en el Patronato José M. ${ }^{a}$ Cuadrado, lograba, al mismo tiempo, el control de su actividad y, lo que tenía gran importancia en el momento, el reconocimiento de la importancia que se otorgaba a su labor al considerarlos dignos de formar parte del organigrama funcional del Consejo Superior de Investigaciones Científicas, aun cuando fuera en el seno de un Patronato y no como Instituto. Adscripción que con gran satisfacción e incluso orgullo ostentan la mayoría de los centros que, en estos momentos, siguen teniendo en el 
Consejo Superior de Investigaciones Científicas el garante de la unión de todos con todos a través de la CECEL.

Los fundadores de los centros normalmente han sido las instituciones y organismos públicos de segundo y tercer orden, es decir, los más cercanos a la realidad de cada tierra, su historia, sus problemas y sus gentes: ayuntamientos, diputaciones, entidades financieras y de ahorro, asociaciones culturales... Cada centro de estudios ha tenido su fundador.

\section{Clases de protectores}

A lo largo de la historia no han variado las características de los protectores de actividades culturales individuales o colectivas que, igualmente han podido ser y son individuales y colectivos $y$, dentro de estas últimas, privadas $u$ oficiales, pero ha sido muy sustancial el cambio visible en cuanto al número de los unos respecto a los otros. Desde la Antigüedad y hasta el siglo XIX, era predominante la figura del mecenas o protector de artistas y hombres de letras. Hoy se han invertido los términos y predominan los organismos colectivos, tanto privados como oficiales, sobre el mecenazgo individual.

La condición de los protectores y mecenas depende del carácter de cada centro $y_{1}$ a su vez, tal condición se define a partir de su naturaleza jurídica, en la que se basa la división en centros públicos y privados. Diferencia que no afecta en nada a su trabajo, aunque puede que sí a las condiciones en que el mismo se desarrolle, pero sí, y de manera decisiva, a otros aspectos, cuales en el caso de los centros públicos son, por ejemplo, los de su limitación o total incapacidad para recibir ayudas y subvenciones de otros organismos, en virtud de la legislación vigente, y el de la disminución de su derecho a formar parte de los órganos de gobierno de determinadas entidades. Aspectos en los que reina una evidente confusión, con origen en las variadas interpretaciones que se aplican, dando como resultado el caso de un centro que tiene representación de máximo derecho en una entidad, en tanto que se le niega en otra equivalente, ambas de la misma ciudad y ámbito de actuación.

Los públicos, entendiendo por tales aquellos que actúan al amparo de organismos de la misma condición, tienen como protectores a los ayuntamientos, diputaciones, gobiernos de las comunidades autónomas y, en algún caso, una universidad. En tanto que los privados carecen de un protector en sentido estricto.

A pesar de que el nombre de centros de estudios locales hace pensar que son los ayuntamientos los principales detentadores del patronazgo de los mismos, éste es ejercido en la mayor parte de los casos por las diputaciones provinciales en lógica relación con el espacio geográfico de actuación de los centros en la que, de acuerdo con lo ya dicho al respecto, hay un claro predominio de los estudios y publicaciones dedicados a provincia sobre los referidos a cualquiera otra división política o administrativa. La identificación entre espacio geográfico y ámbito de estudio es tan preciso en la mayoría de los casos que, igualmente como consecuencia natural, que sean las diputaciones provinciales las que se encarguen del patrocinio de tales trabajos que ha hecho que muchos casos se hayan llegado a considerar como una parcela más dentro de sus obligaciones de atención a las necesidades de la provincia. Siguiendo un camino habitual cual es el de convertir en público y dependiente una tarea independiente, no privada, carácter que no es aplicable con el debido rigor a cualquier actividad que se desarrolla al amparo del apoyo económico y las subvenciones de los organismos oficiales.

La asunción de las obligaciones de patronato por parte de las diputaciones provinciales sobre los centros de estudios locales ha sido objeto de una ampliación de objetivos al asumir su patrocinio las comunidades autónomas uniprovinciales, al mismo tiempo que algunas de las comunidades más amplias las han agrupado en una especie de federación, a semejanza del Instituto de España y las academias nacionales, con el objeto de poner al servicio de la comunidad correspondiente la suma de los estudios y actividades de todos los centros.

Suele haber plena coincidencia entre el fundador y el protector de cada centro, no siendo frecuentes los casos en los que se ha producido un cambio, ya que la principal función, a veces única, es la de hacer posible el mantenimiento de la actividad mediante la financiación de los gastos.

En los casos en los que la protección corre a cargo de ayuntamientos y diputaciones el alcalde o presidente suelen ostentar, como anejo al cargo, el título de patrono o 
protector, siendo una figura de corte más honorífico que ejecutivo, aun cuando en este aspecto debe tenerse en cuenta la personalidad del patrono de turno que, no se olvide, se trata de alguien que ostenta la función de patrono en virtud de su cargo político, con no excesivas obligaciones, pero igualmente con escasa repercusión social y beneficio en el plano político, al ser los centros organizaciones no dedicadas de modo especial a la cultura de masas. Aspectos que hacen que la labor del protector de turno dependa más de su postura personal que de los estatutos y reglamentos que puedan regir los centros de estudios locales.

La situación, nunca general y siempre varia y cambiante, hace que la financiación de los centros adopte diversas formas. En unos casos se emplea el método de la subvención consistente en la entrega de una cuantía fija, que puede adoptar la del pago de los gastos justificados que no excedan de una determinada cuantía, de tal modo que los centros deben atender con dichas cantidades a todos los gastos de mantenimiento de instalaciones, servicios y personal y actividades propias. Apoyo que permite una actuación libre de los centros acogidos a tal sistema, pero que, normalmente, no les permite desarrollar una amplia actividad por lo limitado de las subvenciones.

La variante mixta que supone la financiación de los gastos de instalación y funcionamiento a cargo de la entidad protectora, y de subvención para las actividades, en estos momentos no es fácilmente compatible con la independencia del centro correspondiente, dadas las condiciones legales que se imponen para la concesión de subvenciones.

Desde hace algún tiempo se ha producido un importante cambio en la protección a los CEL, que no es más que la aplicación a los mismos de los nuevos criterios que presiden la que se ejerce sobre otras entidades y grupos de actividades equivalentes, especialmente las de carácter cultural y de atenciones sociales. De este modo la protección directa, incluidas las subvenciones y otras ayudas, se están sustituyendo por, un lado, por la absorción del centro por parte de las entidades protectoras, de tal modo que pasan a formar parte de sus servicios culturales, como ya hemos señalado.

Otra solución utilizada actualmente es la de que el centro dependa exclusivamente de los miembros, socios en sentido estricto, que sin mediación de ningún otro organismo hacen posible su funcionamiento y fijan su actividad. Solución que, en su totalidad, no es fácil conseguir cuando se trata de una asociación exclusivamente cultural, sin añadidos. Por ello, las fórmulas jurídica y organizativa más utilizada es la de la fundación que, en buena parte, es semejante a la figura del patronato, pero más funcional por cuanto es más ágil en su funcionamiento, permite la participación libre de los socios que lo deseen que, además, no adquieren unas obligaciones tan rigurosas como en el patronato y, por último, permiten una mayor libertad de actuación a los centros, a cambio de una merma de la seguridad y un aumento del control administrativo. Caracteristicas exclusivamente organizativas y funcionales, ya que, en general, las fundaciones suelen estar mantenidas por las mismas instituciones y entidades de siempre: ayuntamientos, diputaciones provinciales, cajas de ahorros, grandes empresas...

\section{FinANCIACIÓN}

Los centros de estudios locales, como ocurre con cualquier otra organización de carácter estrictamente cultural, carecen de los medios no ya suficientes, ni siquiera mínimos, para el desarrollo de sus funciones, de tal modo que es imprescindible que no sólo su actuación sino su simple supervivencia necesitan del apoyo de personas y organismos que aporten los medios necesarios bajo la figura del mecenazgo o patronato.

La forma de mecenazgo está poco extendida entre los centros de estudios locales y su alcance actual apenas si pasa de la condición de casos aislados, orientada no a posibilitar el trabajo del centro en cualquiera de sus aspectos, sino como apoyo a una determinada actividad: publicación de un libro, organización de un congreso o un acto de carácter excepcional o, al menos, destacado... Entendiendo por actividad o acto destacado aquel que tiene una amplia repercusión en los medios de comunicación, lo que a su vez explica que los centros de estudios locales no suelen ser frecuentes beneficiarios de ayudas de mecenazgo de cuantía importante, que suelen reservarse a entidades y actuaciones capaces de devolver lo invertido, al menos en parte, aunque, naturalmente, no en dinero. Por ello, aun no siendo fácil ni frecuente, es posible que una Academia nacional o una catedral puedan tener el mecenazgo que no es fácil llegue a una academia provincial o a una iglesia rural. 
La financiación mediante la fórmula de subvenciones es el tipo de patrocinio que ha sido más empleado en el caso de los centros de estudios locales. Sistema que sigue vigente, pero mostrando evidentes sintomas de crisis, por un lado, debido al gran número de asociaciones que aspiran a tales ayudas para sus propios fines $y$, por otro, a la peculiar consideración actual de lo que es la Cultura, origen de un concepto de tal amplitud que en él caben las más variopintas actividades y manifestaciones que, a su vez, pueden ser consideradas desde distintos puntos de vista, de acuerdo con sus postulados y prácticas.

Sin duda, el conjunto de actividades culturales -empleando el término en su más amplio y distendido sentido- muy aceptadas por los diferentes grupos sociales, sobre todo de edad, y fáciles de reconocer son aquellas que, con independencia de su forma, se caracterizan por su constante apelación a la creatividad, lo lúdico, el ocio activo, la interactividad $y$, naturalmente, el uso, conquista y disfrute de una cultura cuyos beneficios debe alcanzar a una gran mayoría, a todos, sin que ello suponga esfuerzo alguno por parte de ninguno de ellos. Características de una cultura y sus correspondientes beneficios que, a lo que podemos todavía ver, nunca formaron parte de las actividades de los miembros de la CECEL. Actividades a las que todo tipo de organismos dedican buena parte de los recursos dedicados al área de su política cultural.

Pero, acaso, el mayor problema a que desde hace algún tiempo se enfrentan los centros de estudios locales Ilegado el momento de obtener subvenciones es el de la asunción de sus funciones precisamente por aquellas entidades que los subvencionan, mediante un proceso que ha adoptado dos formas cuya consecuencia más visible e inmediata es la pérdida de independencia en la decisión de las actividades que deban realizarse ya que, en última instancia, puede ser la entidad correspondiente la que decida lo que convenga.

\section{ÁMBITO TERRITORIAL}

La denominación de centros de estudios locales con la que se denomina en general a todas las instituciones que, además, se subraya en la asociación en que se agrupan la mayoría, CECEL, hace pensar que todos los centros limi- tan su área geográfica de estudio y tratamiento cultural a una sola localidad, criterio que en realidad sólo puede aplicarse a unos pocos, en tanto que la mayoría suelen abarcar ámbitos más extensos. Con independencia de las características funcionales o cualquier otro orden que pueden variar sustancialmente de unos centros a otros, la nota común a todos es el de su especificidad espacial, sea ésta la que fuere, de tal modo que se da el caso de la existencia de instituciones que pueden abarcar uno o más espacios claramente delimitados, sin por ello romper el criterio indicado de especificidad o, si se prefiere, de especialización espacial. La Institución Fernán González tiene definido, incluso en sus estatutos, el interés preferente por los estudios que afecten a la provincia de Burgos, en tanto que el Instituto de Estudios Mirandeses desarroIla una brillante actividad de trabajos y publicaciones en áreas de conocimiento semejantes, pero dentro de ellas con una dedicación preferente, casi específica, al territorio de influencia de Miranda de Ebro, sin que ello implique inconveniente alguno para el correcto funcionamiento de ambas entidades, sino que, al contrario, supone un evidente enriquecimiento, acrecentado día a día. Al igual que ocurre, con la labor realizada en Aranda de Duero y su comarca, aunque en este caso, el organismo que centra la actividad sea la Biblioteca Municipal, que no ha adoptado la figura jurídica de centro de estudios.

En algunos casos el nombre de los centros de estudios, a semejanza de lo que ocurre con su ámbito geográfico, no refleja de manera precisa el alcance de los trabajos que en ellos se realizan, como es fácil comprobar mediante una somera consideración de los contenidos de las publicaciones, periódicas o no, que editan. En general, es normal que los libros y publicaciones no periódicas se dediquen a temas de su especialidad temática y ámbito territorial, línea que con frecuencia se altera en las publicaciones periódicas, con el consiguiente enriquecimiento de los contenidos.

\section{Organización INTERna}

Todos los miembros de los CEL participan, sin excepción, de una decidida vocación por el apoyo en la esfera de

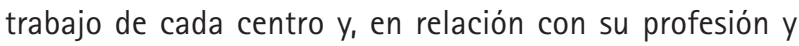
el modo de acceso, la participación personal en la elabo- 
ración y divulgación de dichos trabajos. Modos de participación que se reflejan en la distinta denominación que, siempre de acuerdo con los estatutos, se aplica a cada una de los tipos de miembros: numerarios, asociados, honorarios, correspondientes y otras que, a su vez, justifican las diferentes obligaciones de cada uno con la entidad, siempre de trabajo, ya que los derechos son muy escasos y, siempre y sin excepción, honoríficos y los trabajos sin contrapartida material de ningún tipo, a excepción de los que desarrollan su trabajo como funcionarios de la entidad patrocinadora, normalmente en puestos administrativos.

Los componentes de los CEL se diferencian por el procedimiento seguido en su ingreso en el centro que, de acuerdo con la naturaleza del mismo y según suele especificarse en los estatutos, pueden tener un número indefinido o limitado. En el primer supuesto, puede hablarse de centros abiertos, ya que puede formar parte de los mismos cualquier persona que reúna unas determinadas condiciones que, en ningún caso, resultan excesivas y, precisamente por ello, su contribución es la propia de los socios que pueden reducirse a contribuir con su ayuda económica al funcionamiento del centro. Aportación que pueden completar con su trabajo en los puestos de dirección y organización, a los que todos los miembros tienen derecho a acceder si son propuestos y elegidos para ello.

Los centros formados por un número determinado de miembros son aquellos que adoptaron la organización y funcionamiento interno de las academias $y$, por ello, suelen denominarse como tales, incluso con el título de Real. La diferencia esencial respecto al otro tipo de centros es, como hemos dicho, el número limitado de miembros que, en este caso, son elegidos teniendo en cuenta determinadas condiciones basadas en la titulación académica y, de manera especial, casi decisoria, los trabajos desarrollados y las publicaciones de los mismos, consideradas como aportaciones al conocimiento en el campo de actuación de la academia. El nombramiento lleva consigo, con carácter obligatorio, la elaboración y lectura pública de un trabajo de investigación original o la entrega de una obra, en el caso de artistas plásticos. Trabajo que, normalmente, no es sino el principio de una actividad que desarrollará posteriormente. Aunque, en este punto, son muy varios los conceptos, si bien no tanto como las conductas. Algunos piensan que su elección, siempre debida a sus demostrados méritos, es suficiente para justificar su presencia, sin más obligaciones que asistir a cuanto acto o reunión se celebre y, lógicamente, adaptan su conducta a tal criterio. En este caso se desconoce o se olvida que una academia provincial no es el lugar de acogida de los ilustres del lugar, incluso de los que lo son por otros saberes o sólo de nombre, sino el de acogida de ilustres de los que se espera sigan trabajando a través de él.

No faltan ejemplos de entidades que son, a la vez, genuinos centros de estudios locales y academias provinciales.

En los casos señalados nos referimos siempre a los llamados miembros numerarios que son los que, con independencia del procedimiento de selección, gozan de todos los derechos y deben cumplir las obligaciones que fijan los estatutos y el reglamento de régimen interno de cada

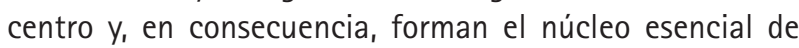
cada centro. A ellos se unen, en calidad de colaboradores y copartícipes una serie de personas que suelen ser seleccionadas en virtud de sus méritos personales y conducta de ayuda y colaboración con el centro, con la denominación de académicos o socios de honor, honorarios, correspondientes, protectores...

\section{Campos de actividad}

Los campos de actividad dentro de las áreas de conocimiento, con independencia del área geográfica, están configuradas en virtud de los medios de todo tipo con que cuentan los centros de estudio locales, siempre limitados y aun cuando son muchos los que cuentan con domicilios dignos e instalaciones simples pero suficientes, es normal que carezcan de instalaciones, utillaje, personal, financiación... imprescindibles para la realización de trabajos científicos experimentales y técnicos, y concentren su actuación en el campo de las ciencias no experimentales y de las hoy llamadas Humanidades, sin detrimento de que en algunos centros se ayude y estimule la actividad en los campos antes señalados mediante la concesión de becas, ayudas y premios a trabajos y estudios de interés para el ámbito territorial correspondiente.

En algunos centros las actividades de investigación y de publicación de los resultados han experimentado un pro- 
fundo cambio debido a la creación de universidades en lugares donde antes no existían, lo que ha ocasionado no un cese de la actividad por parte de los miembros de los centros, sino una especie de ambivalencia por ser muchos los investigadores miembros de ambas entidades, siendo lo más frecuente que la investigación propiamente dicha se realice con preferencia en la universidad, en tanto que no ocurre lo mismo en cuanto a su publicación, aspecto en el que los centros han asumido tareas en las que llegan a superar a la propia universidad, especialmente en el campo de las Ilamadas Humanidades y Ciencias Sociales.

\section{INSTALACIONES}

Es imposible $y$, además, falso hablar de las instalaciones de los CEL en forma general ya que, en este punto, es donde son más apreciables las diferencias. Algunos centros tienen espléndidos domicilios sociales, con dependencias suficientes, capaces y perfectamente equipadas para todos los servicios, con el complemento de salas de actos para el desarrollo de todas las actividades públicas. Domicilios en los que un equipo de personas, en número suficiente y de adecuada preparación realiza los trabajos necesarios para el desarrollo de las actividades.

Más numerosos son los centros que disfrutan de instalaciones que se pueden calificar como suficientes, siempre que se consideren en relación con la actividad del centro, pero $\sin$ excesos, con un personal reducido a una o dos personas, cuya preparación y generosidad en el trabajo les hace polivalentes. Por supuesto, son locales en los que es imposible desarrollar actos públicos.

No faltan los centros cuyo domicilio se reduce a una sola estancia, carente de personal a su exclusivo servicio y sin capacidad para atender todos los días a los posibles solicitantes de sus servicios que, sin embargo, son atendidos por los miembros del centro.

\section{Servicios QUe PRESTAN}

Además de las publicaciones sobre temas propios de su dedicación, periódicas o no, los CEL hacen una intensa labor de divulgación cultural mediante la organización de conferencias, mesas redondas, simposios, congresos y todo tipo de reuniones científicas a cargo de sus miembros y de especialistas invitados. Labor que, en muchos casos, se complementa con conciertos musicales, exposiciones de obras artísticas y de carácter cultural, concursos pictóricos y literarios, concesión de ayudas y becas para la realización de trabajos de investigación sobre temas relacionados con su ámbito de actuación.

No obstante, y a pesar de la indudable importancia de las actividades anteriores que son las que, además, tienen un mayor eco, la verdadera contribución de la mayoría de los CEL a la cultura, en general, y a algunas parcelas del saber, en particular, se realiza a través de las publicaciones, tantas veces citadas, convertidas en fuentes imprescindibles de consulta para todo tipo de personas interesadas en el conocimiento de un tema específico en una localidad o provincia. Servicio que se completa, mediante una labor silenciosa, menos conocida por el gran público, pero muy apreciada por los interesados en la verdadera cultura $y_{\text {, }}$ sobre todo, por los investigadores, mediante las consultas en los fondos bibliográficos y documentales en bibliotecas y archivos, que han perfeccionado sus servicios con los más modernos procedimientos de consulta y reproducción al servicio de todos.

En relación con estos servicios es obligado llamar la atención sobre la importancia de los fondos de las bibliotecas y archivos de los centros que, en gran parte, a excepción de los investigadores y especiales aficionados no son suficientemente conocidos.

\section{Situación Actual}

Los centros de estudios locales conservan sus denominaciones originales y su caracteristica fundamental, que no es otra que la fidelidad al estudio y divulgación del pasado y el presente de la tierra de la que se ocupan, con el mismo entusiasmo de siempre; pero, y en esto encontramos el carácter esencial de su trabajo presente, con menos limitaciones en cuanto a los temas y con más rigor en su tratamiento.

La ampliación de los temas no ha incidido sólo en los propios del campo histórico, sino que muchos centros han in- 
cluido en sus trabajos los pertenecientes a áreas del saber que tienen una singular relación con el ámbito geográfico, de tal modo que hoy es fácil encontrar centros que dedican su atención a temas muy diversos, en una amplia gama que abarca los agrícolas, industriales, sanitarios, económicos...

Dentro de ese amplio campo se aprecia una clara continuidad con la vocación inicial de cada centro en la gran atención prestada a los aspectos culturales más diversos, destacando las peculiaridades de las diferentes manifestaciones en cada lugar, de manera que, sin detrimento del tratamiento específico de cada tema, los nuevos conocimientos se entroncan con los anteriores $y$, a su vez, se entroncan con los obtenidos en el resto de las manifestaciones, formando una suerte de enciclopedia sobre la zona estudiada, publicada bajo denominaciones diversas en las que es evidente el objetivo de exponer con la mayor amplitud posible la realidad de cada territorio a lo largo de su historia, con particular incidencia en el presente y sin que ello suponga un mero trabajo erudito, como evidencia su utilización en la obtención y exposición de argumentos para pedir y aplicar soluciones a determinados problemas $y$, de manera más genérica, atraer la atención y el interés de los de fuera para convertirlos en visitantes.

El cambio anterior en cuanto a la extensión y cuantía de los temas ha sido incluso mayor en lo relacionado con su forma de valorarlos y tratarlos. Era normal que, desde principios hasta las década de los sesenta del siglo pasado, la atención de los estudiosos de los CEL se enfocara de forma predominante al tratamiento de los episodios y personajes históricos o tenidos como tales relacionados con la ciudad o territorio correspondiente, cuyas peripecias eran tratadas y expuestas en un tono en que el lenguaje servía a una concepción gloriosa de cuanto se exponía. Así nació la figura del erudito local, tan minusvalorada como despreciada, que sigue pesando en la consideración de muchos tratadistas de temas locales, que no tienen relación alguna con aquéllos. Consideración de los eruditos locales y de los CEL del pasado que es, a la vez, errónea e injusta en buena parte, ya que se olvida que gracias a los trabajos realizados por ellos, dados a conocer en las revistas y libros publicados por los CEL se pudieron elaborar la mayor parte de las grandes síntesis publicadas en el campo de la historia y del arte.

En virtud de lo dicho podemos afirmar que nos encontramos ante una nueva etapa de la vida de los centros de estudios locales en la que se ha producido no un gran cambio en el camino a seguir, como suele ocurrir, sino un perfeccionamiento en la forma de recorrerlo. Andadura que se desarrolla en los mismos paisajes y con idéntico y siempre renovado acompañamiento que lo hicieron nuestros antecesores en los centros -siempre beneméritos y merecedores de todo elogio-, con la responsable seriedad y el sentido de servicio que nos dejaron como ejemplo de conducta que, entre otras cosas, nos empuja al cambio para hacerlo mejor, siempre mejor como es obligación de todo honrado heredero $y$, por supuesto, para pervivir, no para hacer de nuestra tarea el fruto de una moda o un devaneo más de la corriente lúdica del momento y de las muestras de la cultureta de turno, dando a luz eventos divertidos cual fuegos artificiales, de usar y tirar que, como el personaje de Quevedo, "fuese y no hubo nada". Nuestro pasado merece el máximo respeto y nuestro presente no nos permite dispendios, ante todo, de talento y buen gusto $y$, en segundo plano, incluso de un dinero, elemento accidental del que los centros no sueles andar muy sobrados.

\section{RefLEXIÓN FINAL}

Las instituciones de estudios locales son realidades culturales de las que no existe un conocimiento acorde con su realidad pasada y presente. Además, entre los escasos conocedores es muy elevado el concepto peyorativo sobre las mismas, que con unánime y machacona insistencia se basa en lo que llaman obsolescencia de su organización y funcionamiento y en el escaso valor de sus actuaciones, cuando no se las tacha de simplemente ineficaces.

Juicio nacido, y es de justicia aceptarlo, en el momento en que las academias dejaron de cumplir su misión al adoptar un cierto abandono en el rigor de sus tareas y en la elección de sus miembros, basada en méritos acaso innegables, pero con escasa o nula relación con los fines y tareas académicas, que produjo esporádicos frutos, dentro de un desierto de hechos que, con una especie de peculiar nominalismo, se pretendía hacerlo brillar por los nombres expuestos.

Se inició un estado de opinión que aún padecemos, y lo realmente grave es que se proyecta sobre el trabajo hecho en el pasado y hace que se ignore e infravalore lo que de verdad supone la existencia de academias e instituciones

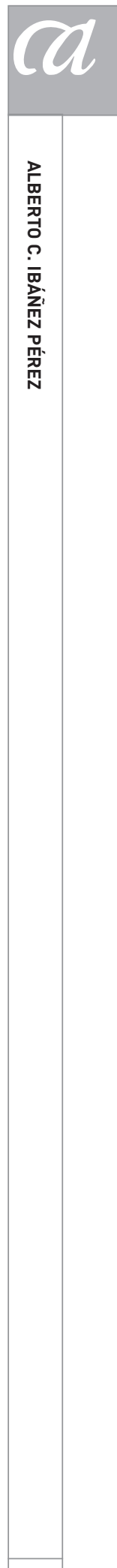


de estudios locales desde hace años para el conocimiento de la diversas muestras de la cultura y de los hombres de nuestra tierra que, en perfecto acuerdo con el tiempo actual, se encargan de custodiar y preservar para el futuro los valores y obras del pasado ya consolidadas por el tiempo y la memoria $y_{1}$ a la vez, cuanto se vaya creando y consolidando en el presente con ideas y creaciones que sean algo más que originales fogonazos que el paso de la moda se llevará consigo. Valores y obras que las instituciones de estudios locales no crean ni destruyen, sólo acogen, conservan, estudian y dan a conocer. Pasado el tiempo, cuando las noticias de prensa, los artículos de los amigos y las gacetillas duermen el sueño de las hemerotecas, los trabajos de los CEL jerarquizan y colocan a cada autor y cada obra en el lugar que les corresponde, pinchando globos, exaltando humildades y rompiendo silencios.

Entre su más visible realidad cuentan las publicaciones y revistas literarias y científicas -incluyendo entre las ciencias la Historia- con colecciones de cientos de números, con cientos de miles de páginas repletas de conocimientos y datos sobre nuestra tierra y sus hombres. Sacados de la lectura de miles de documentos, de libros antiguos, de tradiciones y leyendas oídas a memoriosos habitantes de la llanura, el páramo y la montaña. Todo ello, debidamente jerarquizado, convertido en saberes escritos al alcance de cuántos quieran acercarse a ellos.

Los CEL son autores de una obra elaborada con materiales vivos, sin el cartón piedra de un parque temático y sin mitomanías. Hecha a medida del tiempo, atenta a valorar no lo que dicta la moda, sino lo que muestra su capacidad de perdurar, sin desprecio ni papanatismo de las glorias pasadas y con el adecuado sosiego ante las urgencias del presente. Sin caer en la hispánica veneración por las ruinas y las goteras, que hace incómoda la visión del pasado y el vivir presente, ni derribándolo todo para no correr el riesgo de quedarnos a la intemperie.

En los CEL se hace y se pretende seguir haciendo una historia a mitad de camino entre la respetable nostalgia de algunos, y la agresiva y utilitarista facundia de la modernidad y de la posmodernidad no tan respetable en su carga de "marketing", "vending" y, en fin, foto y "pan para hoy...".

Por lo dicho, creo que es oportuno recordar la conveniencia de que los llamados poderes públicos, se den cuenta de que las academias e instituciones de estudios locales son algo más de lo que los perezosos mentales creen que son $y_{\text {, en }}$ consecuencia, alivien el abandono de muchas de ellas y refuercen el aprecio y la consideración a todas. Merece la pena hacerlo. Basta recordar que sigue siendo cierto que: "Nos fugit Historiae enmondum fulgit iberis", que traducido en libertad nos enseña que "cada vez que sobre lberia fulge la luz de la Historia, la noche se bate en retirada". Aunque, en verdad, debemos recordar que la oscuridad y la noche no se muestran sólo en la ignorancia, sino que hoy, aunque menos visibles, acaso ambas alcancen su mayor espesor en el terreno de los falsos saberes.

Recibido: Octubre de 2007

Aceptado: Mayo de 2008 